\title{
REDESCRIÇÃO DOS TIPOS DE VERONICELLIDAE (MOLLUSCA, GASTROPODA) NEOTROPICAIS. X. OS TIPOS DE DIPLOSOLENODES OCCIDENTALIS (GUILDING, 1825) NO BRITISH MUSEUM (NATURAL HISTORY), LONDRES
}

JOSÉ WILLIBALDO THOMÉ

\section{ABSTRACT}

Diplosolenodes occidentalis (Guilding, 1825) (Mollusca, Gastropoda, Veronicellidae) is redescribed upon the examination of types in the British Museum (Natural History). New characters of external and internal morphology are described. A lectotype is selected.

\section{INTRODUÇÃO}

Em recente viagem a Londres, como Bolsista do "Deutscher Akademische Austauschdienst" (DAAD) da República Federal da Alemanha, fomos surpreendidos com o aparecimento de um lote de lesmas nas coleções em líquido do setor de Moluscos do "British Museum (Natural History)" pertecente originalmente à coleção do Reverendo Lansdown Guilding e rotulado como síntipos da espécie Onchidium occidentale Guilding, 1825. Este lote não fora por nós localizado anteriormente (Thomé, 1979), quando redescrevemos os tipos de Veroniceillidae neotropicais daquela coleção.

Tratando-se de uma espécie das primeiras descritas na Família e cuja identificação tem trazido grandes controvérsias, julgamos que a redescrição, com fixação do lectótipo, será valiosa contribuição para a elucidação da sistemática da família nas Antilhas.

A metodologia empregada é a mesma dos trabalhos anteriores (Thomé, 1969a-b, 1970a-b, 1971, 1972, 1973, 1976, 1979).

\section{Diplosolenodes occidentalis (Guilding, 1825)}

Onchidium occidentale Guilding, 1825: 322, pl. 9, figs. 9-12; Guilding, 1845: 294, pl. 31, figs. 8-11; Thomé, 1975a: 155, 158.

Vaginula occidentalis; Deshayes, 1832: 966, pl. 8E, fig. 10; Fischer, 1871: $150,164,175$, pl. 11, figs. 2-4 (partim); Fischer, 1875: 54; Fischer \& Crosse, 1870-1902: (v. 1) 673, 676, 681, 682; (v. 2) 727, pl. 28, figs. 21-26 (partim); Semper, 1885: 299, 313, pl. 25, fig. 1, pl. 27, figs. 13-14 (partim); Heynemann, 1885a: 4, 16 (partim), 1885b: 274, 278 (partim); Crosse, 1890: 237 (partim); Heynemann, 1906: 70 (partim); Colosi, 1922: 477; Coifman, 1935: 325, 331 (partim).

Vaginulus occidentalis; Shuttleworth, 1854: 126.

Veronicella occidentalis; Bland \& Binney, 1873: 339; Cockerell, 1893: 194; Collinge, 1893: 219; Guppy, 1893: 222 (partim); Cockerell \& Larkin, 1894a: 24, 1894b: 60; Dundee, 1974: 1, 7 (partim).

Cylindrocaulus occidentalis; Hoffmann, 1925: 5, 10, 42, 50, 113, 144, 152, $206,215,217,234,266,272,277,290,296,339,372$, pls. 5-6, f. d2 (partim).

Vaginulus (Latipes) occidentalis; Baker, 1925: 159, 169, 172, 174, 184, pl. 5, figs. 18-20 (partim), 1926: 29 (partim). 
Leidyula occidentalis; Hoffmann, 1927a: 219 (partim), 1927b: 2, 19, 22, 33 (partim).

Vaginulus (Latipes) occidentalis occidentalis; Baker, 1931: 138 (partim).

Diplosolenodes occidentalis (sic); Thomé, 1975b: 14.

\section{Redescrição}

a) Morfologia externa (figs. 5-6).

Animal de tamanho médio, mais largo do que alto, alongado, quase reto (levemente curvado sobre o pé). Noto abaulado; perinoto destacado, sem limitação precisa; hiponotos oblíquos, para trás quase horizontais. Animal algo desbotado, de cor geral beje escura. No noto percebe-se pontuações escuras esparsas, o perinoto é claro e os hiponotos, também claros, deixam perceber pontuações escuras, amplamente esparsas. Sola apenas mais estreita do que o hiponoto da direita, não alcança os sulcos pediosos; sem pigmentação, clara e sem linha longitudinal mediana. Poro genital feminino bem próximo ao sulco pedioso e no meio ou à frente da metade do comprimento do animal. Ânus circular, levemente para a direita, não totalmente encoberto pela sola livre do pé. (Medidas do lectótipo: comprimento $45,0 \mathrm{~mm}$, largura: $15,0 \mathrm{~mm}$, altura: $8,0 \mathrm{~mm}$, largura da sola: $4,4 \mathrm{~mm}$, largura do hiponoto direito: $5,6 \mathrm{~mm}$; distância do poro genital feminino, do sulco: 0,6 mm, da frente: $19,5 \mathrm{~mm}$, de trás: $21,5 \mathrm{~mm}$ ).

b) Morfologia interna (figs. 1-4).

Alça intestinal anterior recoberta por desenvolvido lóbulo da glândula digestiva.

Glândulas salivares com ácinos grandes, no conjunto de aspecto compacto.

$\mathrm{O}$ reto penetra no tegumento junto ao oviduto, mas acha-se invaginado por uma bolsa, que se extroverte levemente do tegumento (fig. 3) e separa o reto do oviduto. (Medidas do lectótipo: diâmetro do reto junto ao tegumento: $0,6 \mathrm{~mm}$; diâmetro externo da bolsa envolvente, junto ao tegumento: $1,5 \mathrm{~mm})$.

Nervos pediosos juntos, paralelos, acolados ao tegumento, extendem-se para trás quase retos, formando uma leve curva para a direita, até logo depois do poro genital, onde afastam-se um do outro em amplo arco até bem no fim da cavidade geral. (Medidas do lectótipo: comprimento total: $27,6 \mathrm{~mm}$; afastados: $14,3 \mathrm{~mm}$; afastamento máximo: $2 \mathrm{~mm}$; encontro da aorta a $3,5 \mathrm{~mm}$ da origem).

Glândula pediosa achatada, solta, alongada, estreitando-se muito levemente para a ponta; reta até cerca da metade do comprimento, quando sofre uma curva mais ou menos acentuada para a esquerda e logo a seguir para a direita. Zona clara-esbranquiçada externa, larga e bem destacada em ambos os lados, até próximo da ponta; zona amarelada interna destacada desde a abertura, estreitando-se até a primeira curva, podendo apresentar nessa porção um leve fendilhamento mediano. Depois da segunda curva a zona amarela alarga-se bruscamente e distende-se com aspecto lanceolado até a ponta da glândula. A cicatriz do vaso sanguíneo acha-se no centro da área alargada da zona amarela, onde esta apresenta aspecto cordiforme, perto da ponta (fig. 4). (Medidas do paralectótipo: comprimento em posição natural: 7,4 $\mathrm{mm}$; distendida: $10,9 \mathrm{~mm}$; largura sobre a abertura: $2,0 \mathrm{~mm}$; largura na região assinalada por uma seta: $1,2 \mathrm{~mm}$ ).

Espermateca ovóide, apoiada sobre o canal, lateralmente, pelo polo maior e prolongando-se para o ducto de ligação, pela extremidade do polo menor. O canal de tamanho mediano é grosso, diferenciado longitudinalmente em dois tipos de tecidos, um liso, duro, mais escuro (o canal propriamente dito); e o outro, enrugado em gomos transversais, aparentando ser glandular. O canal se funde com o oviduto dentro do tegumento, justo antes do poro genital. Ducto de ligação longo, sinuoso. Deferente médio curto, sinuoso; 

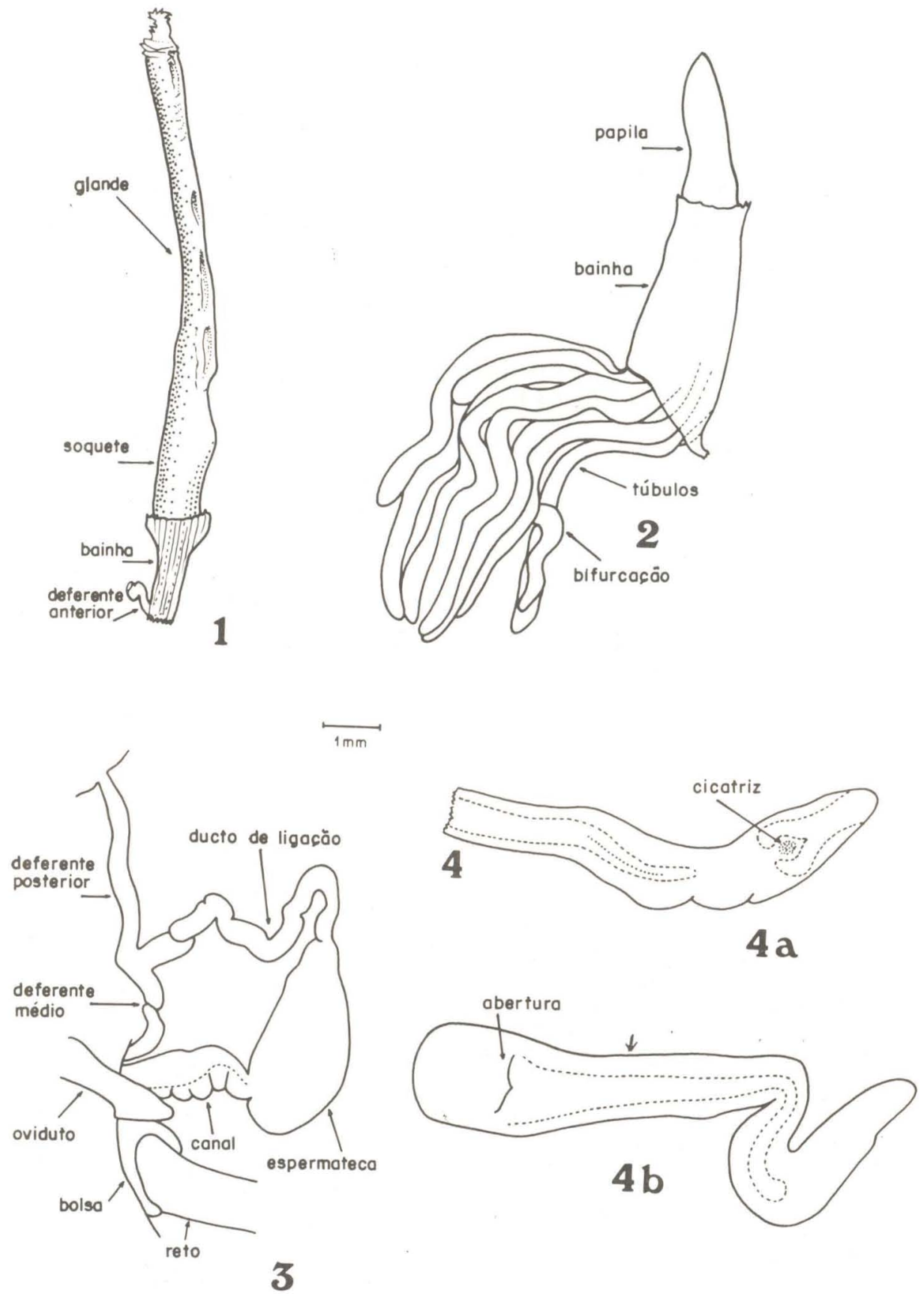

Diplosolenodes occidentalis (Guilding, 1825) (Todas as figuras são do Lectótipo - BMNH 197828W - salvo indicação em contrário). 1. Pênis. 2. Glândula peniana. 3. Espermateca e órgãos junto ao poro genital feminino. 4. Glândula pediosa, vista dorsal, a. lectótipo, b. paralectótipo. 

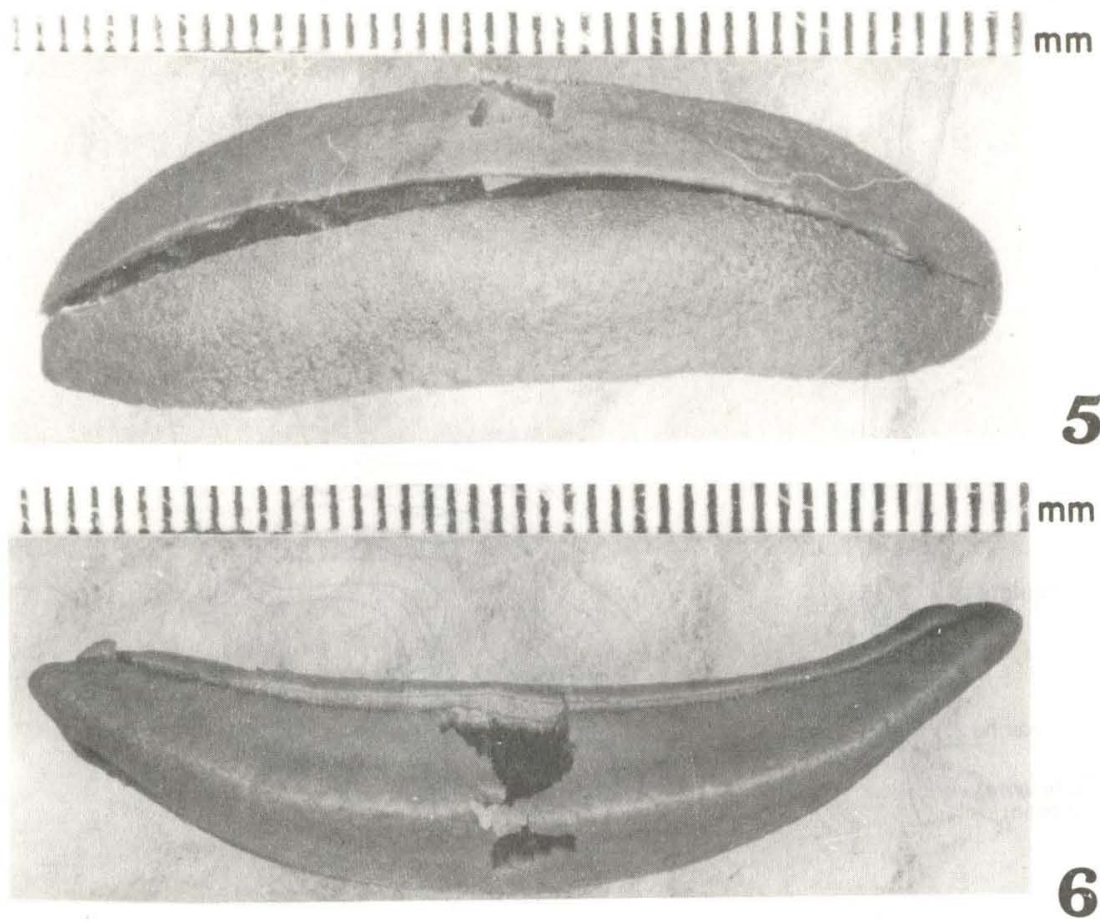

Diplosolenodes occidentalis (Guilding, 1825) (Lectótipo, BMNH 197828W). 5. Vista dorsal. 6. Vista lateral.

penetra no tegumento à esquerda do canal. Deferente posterior de tamanho médio, quase retilíneo (fig. 3). (Medidas do lectótipo: espermateca: comprimento 3,5 mm, maior diâmetro: $0,9 \mathrm{~mm}$; canal: comprimento: $2,0 \mathrm{~mm}$, diâmetro: $0,8 \mathrm{~mm}$; ducto de ligação: comprimento: $5,9 \mathrm{~mm}$, diâmetro: $0,3 \mathrm{~mm}$; deferente médio: comprimento: $1,5 \mathrm{~mm}$, diâmetro: $0,3 \mathrm{~mm}$; deferente posterior: comprimento: $3,4 \mathrm{~mm}$, diâmetro: $0,3 \mathrm{~mm}$ ).

Glândula peniana com papila cônica, alongada, acuminada; possui 16 túbulos com aspecto uniforme, de tamanhos variáveis, 10 longos e 6 curtos, sendo que 3 túbulos apresentam bifurcação, originando, pois, 19 pontas no total (fig. 2). (Medidas do lectótipo: papila: comprimento: 1,6 mm, diâmetro maior: $0,8 \mathrm{~mm}$; túbulos: diâmetros: $0,3 \mathrm{~mm}$, comprimentos: de 5,4 a $6,9 \mathrm{~mm}$ ).

Pênis cilíndrico, alongado, constituído de um soquete curto, liso, que se continua, quase insensivelmente, numa glande algo mais fina e escura, cerca de 6 vezes mais longa do que o soquete; glande com alguns sulcos pouco profundos, em disposição longitudinal, justapostos, desde perto da base até junto à ponta; perto da ponta ocorrem alguns espinhos em fila longitudinal curta e outros circundando a região. A ponta é de um tecido mais mole, afilada, bem espinhosa, sendo que a abertura do deferente é distal (fig. 1). (Medidas do lectótipo: comprimento: $9,5 \mathrm{~mm}$, diâmetro maior: $1,0 \mathrm{~mm}$; comprimento do soquete: $1,6 \mathrm{~mm}$, diâmetro: $1,0 \mathrm{~mm}$; comprimento da glande: $7,9 \mathrm{~mm}$, diâmetro mediano: $0,6 \mathrm{~mm}$ ).

Tipos

Lectótipo: BM(NH), n. ${ }^{\circ}$ 197828W, St. Vincent (Antilhas), "Museum Guilding", designação presente.

Paralectótipo: $\mathrm{BM}(\mathrm{NH})$, n. $^{\mathrm{o}} 19782 \mathrm{Wa}$, mesmos dados do lectótipo. 
Os espécimes nos foram apresentados no mesmo vidro, tendo sido anteriormente dissecados, com corte longitudinal mediano pelo noto e com diversas danificações, que contudo, não impediram que conseguíssemos os dados indispensáveis à redescrição. O material estava identificado por uma etiqueta antiga, colada no vidro externamente, outra etiqueta antiga, danificada, dentro do vidro (nesta há também a referência: "rev. 1884 Heynemann") e uma terceira etiqueta recente, dentro do vidro, com número de registro na coleção.

\section{Observações}

A descrição original, em latim, é sumária e se refere apenas ao colorido geral e mencionava a bifurcação dos tentáculos inferiores. Permitimo-nos a transcrição da mesma: "Onchidium occidentale, dorso fusco atomis brunneis elevatis sparsis, ventre pallido: lateribus livido-maculatis, brachiis apice divisis".

Acrescenta que é freqüente em lugares úmidos elevados da ilha de St. Vincent, onde durante o dia encontram-se contraídas sob pedras. Descreve a postura, que seria de 20 ovos e que o animal recém-eclodido é pálido, com "pescoço" e "cauda" enegrecioios.

Acompanham a descrição acima 4 figuras, representando um espécime adulto, vivo, em posição de rastejamento, visto de cima e ventralmente; um animal recém-eclodido, vista dorsal e uma porção da postura, com 4 ovos.

A reidentificação da espécie, especialmente com material fixado e conservado é deveras precária, tendo em vista os dados da descrição original, o que provocou uma abundante bibliografia sobre a espécie, sem esclarecer em definitivo a sua identificação.

A discussão exaustiva da extensa sinonímia, faremos após exame de material recente topotípico, o qual estamos em vias de receber. (1975b).

A inclusão da espécie no gênero Diplosolenodes é baseada em Thomé

\section{AGRADECIMENTOS}

Ao Dr. Fred Naggs do "British Museum (Natural History)", Londres, que localizou o lote de síntipos na coleção em álcool do Museu e teve a gentileza de colocá-lo à nossa disposição para este trabalho. A minha esposa Clélia pelas fotos, às funcionárias Rejane Rosa pela ultimação dos desenhos e Tânia Carvalho pelos serviços de datilografia.

\section{BIBLIOGRAFIA}

Baker, H. B., 1925. North American Veronicellidae. Proc. Acad. nat. Sci. Philad. 77: 157-84.

Baker, H. B., 1926. Veronicellidae from British Guiana. Ibid. 78: 29-34, pl. 4.

Baker, H. B., 1931. Notes on West Indian Veronicellidae. Nautilus, Philad. 44(4): 131-7, pl. 8.

Bland, T. \& W. G. Binney, 1873. On the lingual dentition and anatomy of Achatinella and other Pulmonata. Ann. Lyc. nat. Hist. N. York 10: 331-51, pls. 13-16.

Cockerell, T. D. A., 1893. A check-list of the slugs. Conchologists, Lond. 2(7/8): 168-196.

Cockerell, T. D. A. \& R. R. Larkin, 1894a. On the Jamaican species of Veronicella. J. Malac., Lond. 3(2): 23-30.

Cockerell, T. D. A. \& R. R. Larkin, 1894b. On the Jamaican species of Veronicella. Nautilus, Philad. 8(5): 60.

Coifmann, I., 1935. Vaginulidi raccolti dal Prof. Beccari nella Guiana inglese. Boll. Zool., Napoli 6(5/6): 325-32, 4 figs.

Collinge, W. E., 1893. Appendix and notes. (para: "A check-list of the slugs"). Conchologists, Lond. 2(8): 196-232.

Colosi, G., 1922. Contributo alla conoscenza anatomica e sistematica dei vaginulidi sud-americani. An. Mus, nac. Hist. nat. B. Aires 31: 475-517, il. 
Crosse, H., 1890. Faune malacologique terrestre et fluviatile de l'Ile de Cuba. J. Conch., Paris 38: 173-335.

Dundee, D. S., 1974. Catalog of introduced molluses of eastern North America (North of Mexico). Sterkiana, Columbus 55: 1-37.

Férussac, D. \& Deshayes, G. P., 1820-1851. Histoire Naturelle générale et particulière des Mollusques terrestres et fluviatiles... Paris, J.-B. Baillière, 2 v., xxiv +868 p., 171 p.

Fischer, P., 1871. Révision des espèces du genre Vaginula Férussac. Nouv. Arch. Mus. Hist. nat., Paris 7: 147-75, est. 11.

Fischer, P., 1875. Supplément à la liste des espèces du genre Vaginula. J. Conch., Paris 23: 53-7.

Fischer, P. \& Crosse, H., 1870-1902. Études sur les mollusques terrestres et fluviatiles du Mexique et du Guatemala. In: Milne-Edwards, M. Recherches Zoologiques pour servir a l'histoire de la faune de l'Amérique Centrale et du Mexique. Séptième partie. Paris, Imprimerie Nationale. v. 1,702 p. v. 2,731 p., 72 est.

Guilding, L., 1825. Description of a new species of Onchidium. Trans. Linn. Soc. Lond. 14(15): 322-4, est. 9, fig. 9-12.

Guilding, L., 1845. Description d'une nouvelle espèce d'Onchidium: In: Chenu, J. C. Transactions de la Société Linnéenne de Londres, Partie Conchyliologique. Tradução do inglês por J. C. Chenu. Paris, A. Franck. 1: 294-5. [Bibliothèque Conchyliologique, 2a. série]

Heynemann, D. F., 1885a. Ueber Vaginula-Arten im British Museum (Natural History) in London. Jb. dtsch. malakozool. Ges., Frankfurt 12: 1-15, est. 1-2.

Heynemann, D. F., 1885b. Die nackten Landpulmonaten des Erdbodens. Ibidem 12: 236-310.

Heynemann, D. F., 1906. Die geographische Verbreitung der Nackschnecken. Eine zusammenfassende kritische Darstellung unserer Kenntnisse derselben zu Anfang des 20. Jahrhundersts. Abh. senckenb. naturforsch. Ges., Frankfurt $30(1 / 2)$ : $1-92,2$ est., 9 mapas.

Hoffmann, H., 1925. Die Vaginuliden. Ein Beitrag zur Kenntnis ihrer Biologie, Anatomie, Systematik, geographischen Verbreitung und Phylogenie. (Fauna et Anatomia ceylanica, III, Nr. 1). Jena. Z. Naturw. 61(1/2): 1-374, est. 1-11.

Hoffmann, H., 1927a. On some North American Vaginulidae. Proc. Acad. nat. Sci. Philad. 79: 209-21, 5 fig.

Hoffmann, H., 1927b. Uber Vaginuliden aus dem Reichsmuseum Stockholm. Ark. Zool., Uppsala 19A(25): 1-39, 15 fig.

Semper, C., 1885. Landmollusèen. In: Reisen im Archipel der Philippinen. Wiesbaden, C. W. Kreidel. Parte 2, v. 3, caderno 7, p. 291-327, est. 24-7.

Shuttleworth, R. J., 1854. Diagnose neuer Mollusken. n. ${ }^{\circ} 6$ - Beitraege zur naeheren Kenntniss der Land- und Suessawasser-Mollusken der Insel Portorico. Bern. Mitth.:125-148.

Thomé, J. W., 1969a. Redescrição dos tipos de Veronicellidae (Mollusca, Gastropoda) neotropicais: I. Espécies depositadas no "Zoologisches Museum" de Kiel, Alemanha. Iheringia (Zool.), Porto Alegre 37: 101-11, 21 fig.

Thomé, J. W., 1969b. Erneute Beschreibung neotropischer Veronicellidae-Typen (Mollusca, Gastropoda). II. Arten aus der Sammlung des SenckenbergMuseums in Frankfurt. a.M. Arch. MolluskenKde, Frankfurt 99(5/6): 331-63, est. 6-13.

Thomé, J. W., 1970a. Redescrição dos tipos de Veronicellidae (Mollusca, Gastropoda) neotropicais: III. Espécies depositadas no "II. Zoologisches Institut und Museum der Universitaet" de Goettingen, Alemanha. Iheringia (Zool.), Porto Alegre 38: 73-88, 28 figs.

Thomé, J. W., 1970b. Redescrição dos tipos de Veronicellidae (Mollusca, Gastropoda) neotropicais: V. Espécies depositadas no "Museo ed Istituto di Zoologia Sistematica della Università" de Turim, Itália. Ibidem 39: 19-31, 17 figs. 
Thomé, J. W., 1971. Redescrição dos tipos de Veronicellidae (Mollusca, Gastropoda) neotropicais: VII. Espécies depositadas no "Muséum National d'Histoire Naturelle", Paris, França. Ibidem 40: 27-52, 22 fig.

Thomé, J. W., 1972. Redescrição dos tipos de Veronicellidae (Mollusca, Gastropoda) neotropicais: VIII. Espécies depositadas no "Institut für Spezielle Zoologie und zoologisches Museum" de Berlim, Alemanha Oriental. Archos. Zool. S Paulo 21(5): 235-81.

Thomé, J. W., 1973. Erneute Beschreibung neotropischer Veronicellidae-Typen (Mollusca, Gastropoda). VI. Arten aus der Sammlung des Universitetets Zoologiske Museum in Kopenhagen, Dänemark. Steenstrupia, Kopenhagen 4(4): 31-50.

Thomé, J. W., 1975a. Estado atual da sistemática dos veronicelídeos americanos. Arqos. Mus. nac. Rio de J 55: 155-65.

Thomé, J. W., 1975b. Os gêneros da família Veronicellidae nas Américas (Mollusca, Gastropoda). Iheringia (Zool.), Porto Alegre 48: 3-56.

Thomé, J. W., 1979. A redescription of types of six species of Neotropical Veronicellidae (Mollusca; Gastropoda) in the British Museum (Natural History). Bull. Br. Mus. nat. Hist. (Zool.), Lond 35(2): 135-146.

Thomé, J. W. \& V. L. L. Pitoni, 1976. Redescrição dos tipos de Veronicellidae (Mollusca, Gastropoda): espécies no "National Museum of Natural History, Smithsonian Institution", Washington, EUA. Revta bras. Biol., Rio de J 36(3): 709-14. 